\title{
The Stellar Wind From the Central Star of NGC 7009
}

\author{
G. Sonneborn ${ }^{1}$, R. C. Iping ${ }^{1,2}$, D. L. Massa ${ }^{1,3}$, and Y-H Chu ${ }^{4}$ \\ ${ }^{1}$ Lab. for Observational Cosmology, Code 665, NASA/GSFC, Greenbelt, MD 20771 USA \\ email: george.sonneborn@nasa.gov \\ ${ }^{2}$ Dept. of Physics, Catholic University of America, Washington, DC 20064 USA \\ ${ }^{3}$ SGT, Inc., Greenbelt, MD 20770 USA \\ ${ }^{4}$ Dept. of Astronomy, University of Illinois, Urbana, IL 61801 USA
}

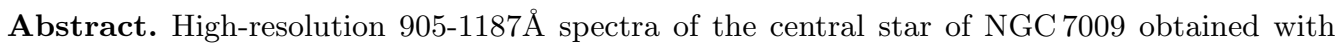
the FUSE satellite provide new constraints on the mass loss and atmosphere parameters. The most prominent spectral feature is a very strong P-Cygni profile of O VI 1032-1038. The only other wind lines are S VI 933-44 (FUSE) and N V 1238-42 and O V 1371 from IUE spectra. C IV 1548-50 and P V 1118-28 are not present, indicating that the wind is very highly ionized. A lower limit on the mass loss rate $\left(\dot{M}>10^{-8} M_{\odot \mathrm{y}^{-1}}, v_{\infty}=2450 \mathrm{~km} \mathrm{~s}^{-1}\right)$ has been derived from an SEI analysis of these wind lines. Preliminary identification of several photospheric spectral features include several strong Fe VII and O VI lines between 1100 and $1170 \AA$ A.
\end{abstract}

Keywords. planetary nebulae: individual (NGC 7009), ultraviolet: stars, techniques: spectroscopic, space vehicles: FUSE, stars: mass loss

NGC 7009 is a classical elliptical PN with a jet-like structure and low-ionization knots on its major axis. High-excitation emission dominates the inner regions of the nebula, indicating a fast-wind interaction may be important. The current parameters of the hot, H-rich central star $\left(T_{\text {eff }}=82,000 \mathrm{~K}, \log \mathrm{g}=4.80\right.$, and $y=N(H e) /[N(H)+N(H e)]=$ 0.08) are from Méndez et al. (1988). UV resonance transitions are the best diagnostics of stellar wind parameters.

NGC 7009 was observed by the Far Ultraviolet Spectroscopic Explorer (FUSE) satellite (Moos et al. 2000), covering 910-1187 $\AA$ at a resolution of $\sim 15 \mathrm{~km} \mathrm{~s}^{-1}$. These data, plus archival IUE spectra (1150-2000A), show that only four stellar wind lines are detected in NGC 7009. These are the resonance doublets O VI 1032-38, S VI 933-44, and N V 1238-42, and the excited state transition O V 1371. The lower level of the O V 1371 line is the upper level of the O V resonance line at $629.73 \AA$. C IV $1548-50$ and P V 1118-28 are not present, indicating that the wind is very highly ionized.

Fig. 1 shows part of the FUSE spectrum of NGC 7009. Preliminary identification of photospheric lines include Fe VII, Fe VI, O VI, and possibly F VI 1139.5 (Werner et al. 2005). Some features are not yet identified. The line IDs are consistent with the stellar radial velocity of $-46 \mathrm{~km} \mathrm{~s}^{-1}$. The width of the stellar features indicates $v \sin i \sim 100 \mathrm{~km}$ $\mathrm{s}^{-1}$. Comparison with the $\mathrm{sdO}$ star BD $+284211\left(T_{\text {eff }}=82,000 \mathrm{~K}, \log \mathrm{g}=6.2\right)$ indicates that Fe VII and O VI, compared with O IV and Fe VI in BD+28 4211, are unexpectedly strong, raising the possibility that $T_{\text {eff }}$ may be underestimated in NGC 7009. Non-LTE modelling of the FUV stellar spectrum is in progress.

An SEI analysis of the O VI, O V, N V, and S VI line profiles was made, following the same procedures used by Massa et al. (2003). From this analysis the terminal velocity in the wind is $v_{\infty} \sim 2450 \mathrm{~km} \mathrm{~s}^{-1}$. The wind law parameter $\beta$ is 0.8 . Model profile fits for these four lines are shown in Fig. 2a. Fig. 2b shows $\dot{M} q$ as a function of $w=v / v_{\infty}$, where 


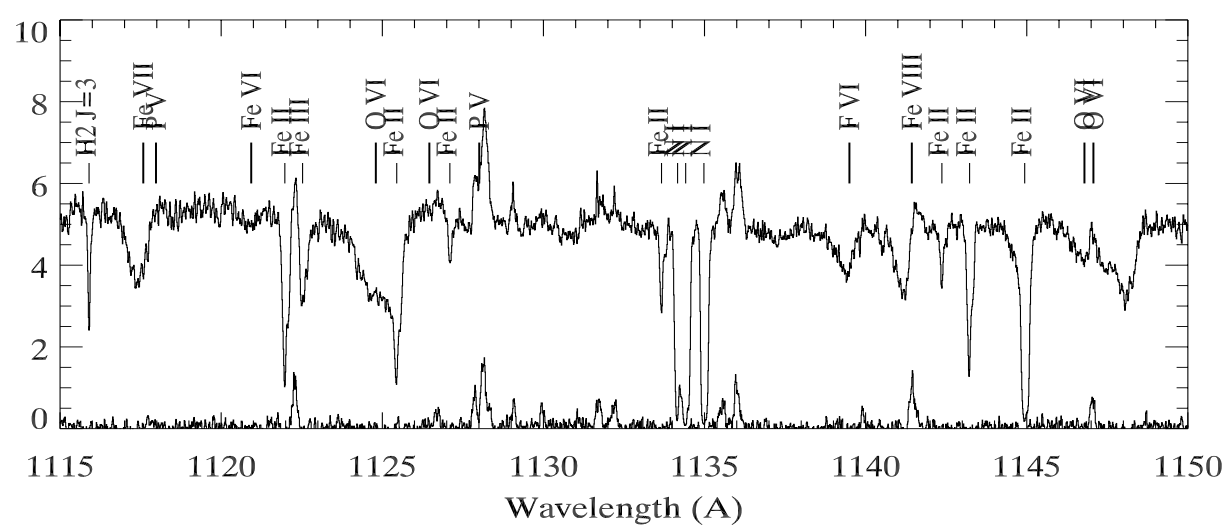

Figure 1. FUSE spectrum of NGC 7009. Line IDs with short tick marks (low ionization) are interstellar; long tick marks (high ionization states) are stellar. A FUSE narrow-slit spectrum of the nebula 3" from the star is at the bottom to show the nebular emission features.

$q$ is the ionization fraction of the ion in question. The O VI line is strongly saturated $(\tau \sim 50)$, and so provides only a lower limit to the mass loss rate $\left(\dot{M}>10^{-8} M_{\odot} \mathrm{y}^{-1}\right)$. The other wind lines are unsaturated and the low values of $\dot{M} q$ for N V and S VI shows that these ions are trace species in the wind.
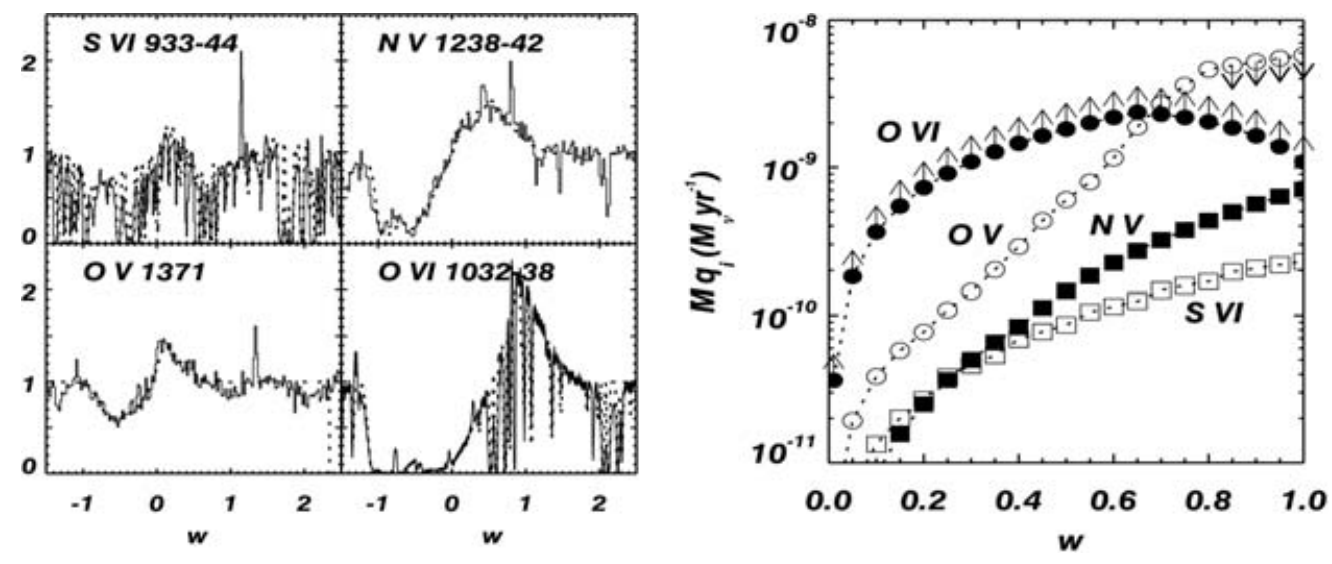

Figure 2. (left) SEI model fits to O VI, S VI, N V, and O V lines for $v_{\infty}=2450 \mathrm{~km} \mathrm{~s}^{-1}$ and $\beta=0.8$. The narrow absorption lines surrounding the S VI and O VI lines are from the Lyman and Werner bands of interstellar $\mathrm{H}_{2}$. (right) Mass-loss parameter $\dot{M} q$ as a function of normalized wind velocity for the O VI, O V, N V, and S VI lines.

\section{Acknowledgements}

The FUSE observations were obtained under programs C176 and X014. This work has been supported in part by NASA grant NAG5-12347 to Catholic University of America.

\section{References}

Massa, D.L., Fullerton, A.W., Sonneborn, G., \& Hutchings, J.B. 2003, ApJ 586, 996

Méndez, R.H., Kudritzki, R.P., Herrero, A., Husfeld, D., \& Groth, H.G. 1988, A\&SA 190, 113

Moos, H. W., et al. 2000, ApJ 538, L1

Werner, K., Rauch, T., \& Kruk, J.W. 2005, A\&\&A 433, 641 